\title{
Intertwined charge, spin, and pairing orders in doped iron ladders
}

\author{
Bradraj Pandey ${ }^{1,2}$, Rahul Soni ${ }^{1,2}$, Ling-Fang $\operatorname{Lin}^{1}$, Gonzalo Alvarez ${ }^{3}$, and Elbio Dagotto ${ }^{1,2}$ \\ ${ }^{1}$ Department of Physics and Astronomy, University of Tennessee, Knoxville, Tennessee 37996, USA \\ ${ }^{2}$ Materials Science and Technology Division, Oak Ridge National Laboratory, Oak Ridge, Tennessee 37831, USA \\ ${ }^{3}$ Computational Sciences \& Engineering Division and Center for Nanophase Materials Sciences, \\ Oak Ridge National Laboratory, Oak Ridge, Tennessee 37831, USA
}

\begin{abstract}
Motivated by recent experimental progress on iron-based ladder compounds, we study the doped two-orbital Hubbard model for the two-leg ladder $\mathrm{BaFe}_{2} \mathrm{~S}_{3}$. The model is constructed by using $a b$ initio hopping parameters and the ground state properties are investigated using the density matrix renormalization group method. We show that the $(\pi, 0)$ magnetic ordering at half filling, with ferromagnetic rungs and antiferromagnetic legs, becomes incommensurate upon hole doping. Moreover, depending on the strength of the Hubbard $U$ coupling, other magnetic patterns, such as $(0, \pi)$, are also stabilized. We found that the binding energy for two holes becomes negative for intermediate Hubbard interaction strength, indicating hole pairing. Due to the crystal-field split among orbitals, the holes primarily reside in one orbital, with the other one remaining halffilled. This resembles orbital selective Mott states. The formation of tight hole pairs continues with increasing hole density, as long as the magnetic order remains antiferromagnetic in one direction. The study of pair-pair correlations indicates the dominance of the intra-orbital spin-singlet channel, as opposed to other pairing channels. Although in a range of hole doping pairing correlations decay slowly, our results can also be interpreted as corresponding to a charge-density-wave made of pairs, a precursor of eventual superconductivity after interladder couplings are included. Such scenario of intertwined orders has been extensively discussed before in the cuprates, and our results suggest a similar physics could exist in ladder iron-based superconductors. Finally, we also show that a robust Hund's coupling is needed for pairing to occur.
\end{abstract}

\section{INTRODUCTION}

The study of iron-based high critical temperature superconductors continues attracting considerable attention in the Condensed Matter community [1-11]. The parent compounds of iron-based superconductors exhibit non-trivial magnetic ordering [12-14] and can have either metallic or insulating characteristics [8-11]. Early theoretical studies based on weak-coupling theory suggested that antiferromagnetic (AFM) order is stabilized by Fermi surface nesting [15, 16], leading to the prediction of superconductivity with $s^{ \pm}$paring symmetry induced by AFM fluctuations $[17,18]$. However, the absence of hole pockets in some compounds $[10,19]$ and the presence of robust local magnetic moments [20] indicated that the role of electronic correlations can not be neglected [11, 13, 14, 21-23]. Superconductivity can be induced in iron-based compounds by either electron or hole doping $[9,17]$ or also by applying pressure [24, 25]. For example, the iron-based compound $\mathrm{BaFe}_{2} \mathrm{As}_{2}$ becomes superconducting by electron doping, namely by partially replacing Fe by Ni [4]. Also superconductivity has been induced in the hole doped $\mathrm{Ba}_{1-x} \mathrm{~K}_{x} \mathrm{Fe}_{2} \mathrm{As}_{2}$ [26] and it can even survive the hole overdoped regime [27]. Interestingly, by hole doping $\mathrm{BaFe}_{2} \mathrm{As}_{2}$, the superconducting transition temperature $T_{c}$ can reach higher values than by electron doping [28].

More interesting for our present study, pressureinduced superconductivity has been achieved in geometries different from planes, namely in the iron-based ladder material $\mathrm{BaFe}_{2} \mathrm{~S}_{3}[24,25]$. In particular, $\mathrm{BaFe}_{2} \mathrm{~S}_{3}$ be- comes superconducting at pressures above $10 \mathrm{GPa}$ with critical temperature $T_{c}=24 \mathrm{~K}$ [24]. At ambient pressure this material is a Mott insulator with stripe-type arrangement, where magnetic moments align ferromagnetically along the ladder rung direction and coupled antiferromagnetically along the legs of the ladder [25]. This exciting experimental progress in quasi one-dimensional iron-based compounds provides a promising platform to explore the magnetism and superconductivity in ironbased materials [24, 25, 29-36] particularly from a theoretical perspective. In particular, due to the availability of powerful numerical many-body techniques for quasione dimensional systems, it is possible to address fairly accurately the ground state properties of iron-based ladder compounds using complex two-orbital Hubbard models incorporating quantum fluctuations, without resorting to crude many-body approximations [37-40].

Already exotic theoretical predictions for iron ladders have been confirmed experimentally. For example, using inelastic neutron diffraction applied to powder $\mathrm{BaFe}_{2} \mathrm{Se}_{3}$, an exotic block-AFM state (involving blocks of $2 \times 2$ iron atoms ferromagnetically aligned, coupled anti-ferromagnetically along the leg direction) was observed after theory predicted such a state [29, 31, 41, 42]. The compound $\mathrm{BaFe}_{2} \mathrm{Se}_{3}$ shows insulating behavior, with energy gap $\Delta \sim 0.13 \mathrm{eV}$ to $0.178 \mathrm{eV}[41,42]$. This compound displays long-range antiferromagnetic order at $\sim 250 \mathrm{~K}$, presumably from interladder coupling, with large individual magnetic moments $2.8 \mu_{B}[29,30,41]$. Interestingly another compound where $\mathrm{K}$ replaces $\mathrm{Ba}$, i.e. $\mathrm{KF}_{2} \mathrm{Se}_{3}$, shows a magnetic arrangement with ferro- 
magnetic rungs coupled anti-ferromagnetically along the legs [43].

Because the work in the related field of computational studies of superconducting $\mathrm{Cu}$-oxides ladders was extremely useful in showing that pairing can emerge from repulsive interactions [24, 44-47], this provides additional motivation to proceed with the numerical studies of iron-based ladder models as well. Experimental work has shown that both $\mathrm{Fe}-$ and $\mathrm{Cu}$-based compounds induce superconductivity via a magnetic pairing mechanism $[13,17,21]$. However, technically there is a practical difference. While $\mathrm{Cu}$-oxide ladders can be described by a one-orbital Hubbard $U$ model [48], the Fe-based ladders require a multiorbital Hubbard $U$ description that must also include the Hund coupling [21]. Increasing the number of orbitals quickly increases the computational effort. However, the competition between charge, spin, and orbital degrees of freedom in Fe-based compounds can also lead to various exotic novel phases with insulating or metallic ground states $[13,21]$.

In the present publication, we study the magnetic and pairing properties of hole-doped two-orbital Hubbard model using the density matrix renormalization group method (DMRG) [49]. For our DMRG calculations, we use realistic hopping parameters for the compound $\mathrm{BaFe}_{2} \mathrm{~S}_{3}$, originally derived in Ref. [40] using $a b$ initio calculations. In a previous work by some of us [40], indications of hole pairing were shown using cluster sizes $L=2 \times 8$. Moreover, the magnetic ordering of the undoped compounds involving FM rung and AFM leg was observed, as in neutron scattering experiments for $\mathrm{BaFe}_{2} \mathrm{~S}_{3}$ [25]. However, in those early calculations the DMRG accuracy with regards to number of states was limited. The present study moves considerably beyond our previous accuracy by using more modern DMRG codes and computational facilities. Now the cluster size $L=2 \times 12$ is reachable. Even more importantly, considerable progress in the physics is here reported.

In particular, we explore the magnetic properties for various values of hole doping and varying interaction strengths over broad ranges. We have observed for the first time that the magnetic order could evolve from the canonical $(\pi, 0)$ order to a more exotic $(0, \pi)$ state, with AFM rungs and FM legs. We have also carefully analyzed the real-space charge density. The number of minima in this quantity is always half the number of holes, suggesting hole pairing, as along as the magnetic background remains antiferromagnetic of any kind. The pairs are arranged in what resembles a charge density wave made of pairs. Remarkably, in regimes where there is no AFM order, such as in fully ferromagnetic regions at large hole doping and Hubbard strength, pairing disappears. Moreover, we study pair-pair correlations and find regions where pairing is robust (in our previous study [40] pair-pair correlations were not addressed). Here, we have calculated both singlet and triplet pair-correlations using operators defined along the rungs of the ladder, and for various hole concentrations. We also study pair correlations employing operators along plaquette diagonals, a hole configuration also prominent in the two-hole bound state. While the rung operator correlations dominate, the diagonal plaquette correlations are similar.

Due to the simultaneous presence of a charge density profile and robust pair-pair singlet correlations, both in a nontrivial magnetic background, we believe our results can be interpreted in a similar manner as recent efforts in the context of cuprates within the umbrella of intertwined order parameters $[50,51]$. In particular, a recent scenario in the context of cuprates proposed by Tranquada [52] expresses that latent tendencies toward superconductivity in individual ladders could lead to an emergent global superconducting state by anti-phase Josephson coupling. While the anti-phase nature of the coupling is needed in $d$-wave superconductors, in our case simply a mere interladder coupling may be sufficient for the entire ensemble to become superconducting. Thus, our results raise the exciting possibility that intertwined order could also be of relevance in iron superconductors, a novel concept.

The organization of the manuscript is as follows. Section II contains the two-orbital Hubbard model employed here for two-leg ladders as well as details of the numerical methods used. Section III starts presenting the DMRG results with focus on the various magnetic orders upon hole doping, as well as the presence of bound states for the case of two holes. Section IV deals with the pair correlations at various dopings and the real-space hole distributions, presenting the idea that intertwined orders could be of relevance for isolated ladders, potentially leading to superconductivity in an ensemble of weakly coupled ladders. Finally, in Section V we present our conclusions.

\section{TWO-ORBITAL HUBBARD MODEL AND METHODS}

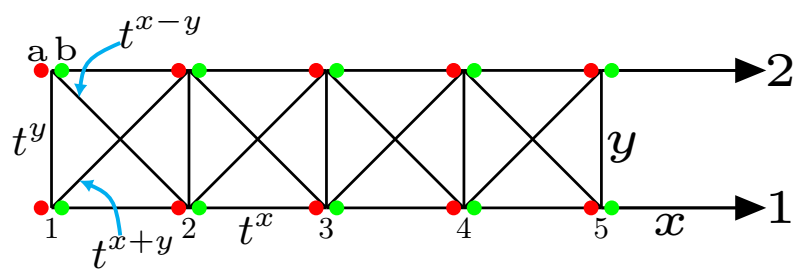

FIG. 1. Schematic representation of a two-leg ladder and the two-orbitals Hubbard model used in this work. At each site, the red circle represents orbital $a$ and the green circle orbital $b$. The $2 \times 2$ hopping matrix along legs is indicated by $t^{x}$ and along rung by $t^{y}$. We have also considered plaquette diagonal hopping matrices $t^{x+y}$ and $t^{x-y}$. For details see text.

In this section, we describe the multiorbital Hubbard 
model for the iron-based ladder compound $\mathrm{BaFe}_{2} \mathrm{~S}_{3}$ used in this work. The multiorbital Hubbard model for a twoleg ladder can be written as the sum of kinetic and interaction energy terms $H=H_{k}+H_{i n}$ [37]. The kinetic portion contains the nearest-neighbor hopping along the leg and rung directions, and also next-nearest-neighbor hopping along the plaquette diagonals of the two-leg ladder. Following the convention employed in Ref. [40], the tight binding term is:

$$
H_{k}=\sum_{i, \sigma, \gamma, \gamma^{\prime}, \vec{\alpha}} t_{\gamma, \gamma^{\prime}}^{\vec{\alpha}}\left(c_{i \sigma, \gamma}^{\dagger} c_{i+\vec{\alpha}, \sigma, \gamma^{\prime}}+H . c .\right)+\sum_{i, \gamma \sigma} \Delta_{\gamma} n_{i, \sigma, \gamma}
$$

where $t_{\gamma, \gamma^{\prime}}^{\vec{\alpha}}$ is the hopping matrix along the directions $\vec{\alpha}$ indicated in Fig. 1 and the orbital space includes two orbitals $\gamma=\left\{d_{x^{2}-y^{2}}, d_{x z}\right\}$. For notation simplicity, these two orbitals will be denoted as $\gamma=\{a, b\}$, respectively. $\Delta_{\gamma}$ denotes the crystal field splitting of those two orbitals at $P=12.36 \mathrm{GPa}$. $n_{i, \sigma, \gamma}$ represents the orbital-resolved number operator at site $i$. For our numerical calculations, we used the same hopping matrices $t_{\gamma, \gamma^{\prime}}^{\vec{\alpha}}$ introduced in Ref. [40]. The actual values of $t_{\gamma, \gamma^{\prime}}^{\vec{\alpha}}$ were obtained from fitting the tight-binding bands with the ab initio downfolded band structure calculations [40]. The $2 \times 2$ hopping matrix between sites $i$ and $i+\hat{x}$ along the legs of the ladder $t_{\gamma, \gamma^{\prime}}^{x}$ is given by (in $\mathrm{eV}$ units):

$$
t_{\gamma, \gamma^{\prime}}^{x}=\left[\begin{array}{ll}
-0.334 & -0.177 \\
+0.177 & +0.212
\end{array}\right]
$$

where $\gamma$ is the orbital index for site $i$ and $\gamma^{\prime}$ for $i+\hat{x}$. $t_{\gamma, \gamma^{\prime}}^{y}$ is the $2 \times 2$ hopping matrix along the vertical rungdirection:

$$
t_{\gamma, \gamma^{\prime}}^{y}=\left[\begin{array}{cc}
-0.024 & 0.000 \\
0.000 & +0.216
\end{array}\right]
$$

The $t^{x+y}$ and $t^{x-y}$ are $2 \times 2$ hopping matrices along the plaquette diagonals of the ladder:

$$
t_{\gamma, \gamma^{\prime}}^{x+y}=t_{\gamma, \gamma^{\prime}}^{x-y}=\left[\begin{array}{ll}
+0.085 & +0.216 \\
-0.216 & +0.109
\end{array}\right]
$$

The crystal fields $\Delta_{\gamma}$ at $P=12.36 \mathrm{GPa}$ for each orbital are $\Delta_{a}=0.423 \mathrm{eV}$ and $\Delta_{b}=-0.314 \mathrm{eV}$. The kinetic energy bandwidth is $W=2.2533 \mathrm{eV}$.

The electronic interaction portion of the Hamiltonian is:

$$
\begin{array}{r}
H_{i n}=U \sum_{i \gamma} n_{i \uparrow \gamma} n_{i \downarrow \gamma}+\left(U^{\prime}-\frac{J_{H}}{2}\right) \sum_{i, \gamma<\gamma^{\prime}} n_{i \gamma} n_{i \gamma^{\prime}} \\
-2 J_{H} \sum_{i, \gamma<\gamma^{\prime}} \mathbf{S}_{i, \gamma} \cdot \mathbf{S}_{i, \gamma^{\prime}}+J_{H} \sum_{i, \gamma<\gamma^{\prime}}\left(P_{i \gamma}^{+} P_{i \gamma^{\prime}}+H . c .\right) .
\end{array}
$$

The first term is the standard on-site Hubbard repulsion between $\uparrow$ and $\downarrow$ electrons in the same orbital. The second term contain the on-site electronic repulsion between electrons at different orbitals and same site. As often employed in previous publications, the standard relation $U^{\prime}=U-2 J_{H}$ is here assumed, due to the $S U(2)$ symmetry of the Hamiltonian. Also the widely employed ratio $J_{H} / U=0.25$ is here also used because iron superconductors are known to have a relatively large Hund coupling. The third term is the ferromagnetic Hund's interaction between electrons occupying the active two orbitals $\gamma=\left\{d_{x^{2}-y^{2}}, d_{x z}\right\}$. The operator $\mathbf{S}_{i, \gamma}$ is the total spin of orbital $\gamma$ at site $i$. The last term is the pair-hopping between different orbitals at site $i$, where $P_{i \gamma}=c_{i \downarrow \gamma} c_{i \uparrow \gamma}$. This pair-hopping term arises from Coulomb interaction matrix elements and has no influence on the more extended pairing of holes due to magnetic short-range order discussed in this publication. Moreover, to confirm this perspective, in many examples we turned off the on-site pair hopping term in the Hamiltonian and our results were barely modified.

To solve this two-orbital Hubbard model for the ladder compound $\mathrm{BaFe}_{2} \mathrm{~S}_{3}$ we employed the DMRG method. Here we focused on a cluster of size $L=2 \times 12$ and with various values for the hole doping. The number of holes $N_{h}$ was obtained by removing $N_{h}$ electrons from the half-filled system with $N=48$ electrons (i.e. 24 sites and 2 electrons per site due to the two orbitals). Due to recent improvement in our computational capabilities, we are able to keep up to $m=3800$ states for our DMRG calculation. As a result we can perform the DMRG calculation quite accurately, with various hole doping concentrations employed for the $L=2 \times 12$ cluster. To characterize magnetic and superconducting properties, we have calculated various observables, such as the charge and spin correlations, spin structure factors, and the pair-pair correlations. For the DMRG calculations we employed open-boundary conditions and we used the DMRG++ software [53].

\section{MAGNETIC ORDER IN DOPED LADDERS}

\section{A. Effect of doping on magnetic ordering at intermediate Hubbard coupling}

For iron-based high- $T_{c}$ compounds, it is widely believed that magnetic-fluctuations play a crucial role to induce superconductivity [21, 48]. For this reason, it is important to determine the magnetic ordering varying the hole doping, starting with the undoped state. At halffilling $\mathrm{BaFe}_{2} \mathrm{~S}_{3}$ is a Mott insulator and displays stripetype magnetic ordering, namely antiferromagnetic order along the legs and ferromagnetic along the rungs [25]. When changing the coupling values and hole concentrations [54], there are possibilities of various types of magnetic ordering, due to the competition between the kinetic energy of holes, the superexchange between local Fe moments, and the Hund coupling. To find the mag- 

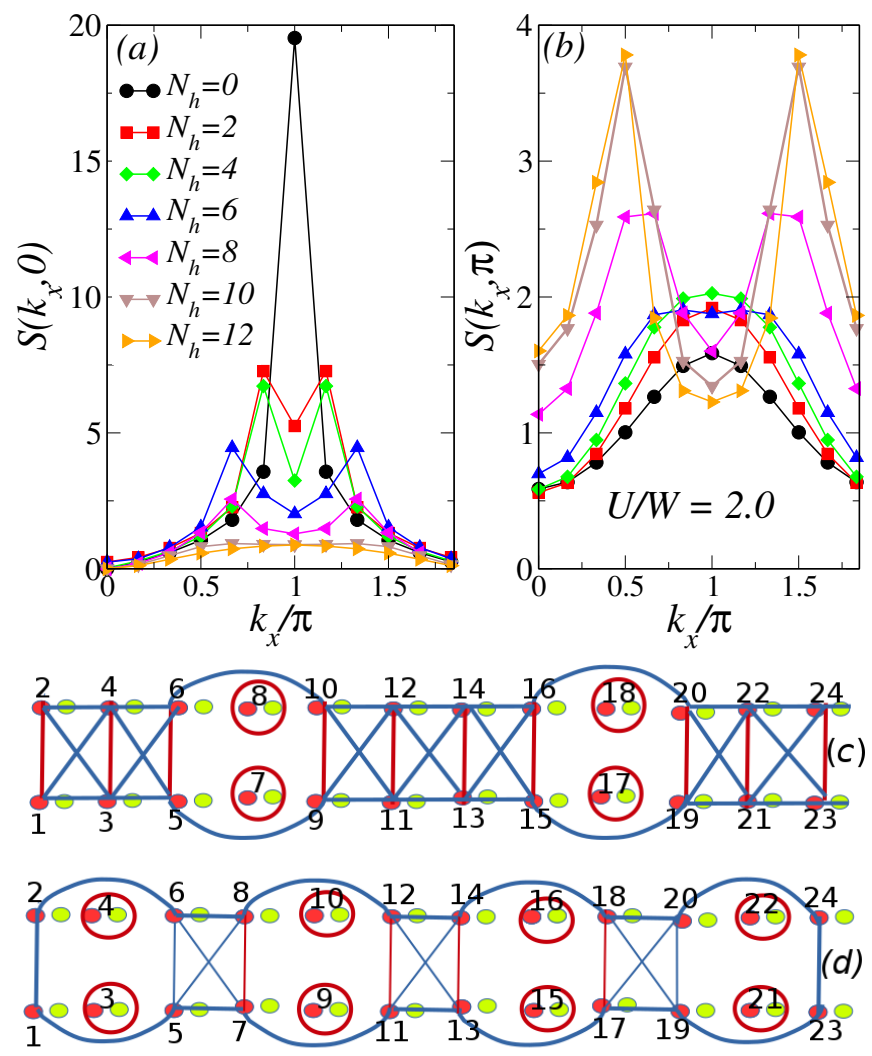

FIG. 2. Spin structure factors (a) $\mathrm{S}\left(k_{x}, 0\right)$ (b) $\mathrm{S}\left(k_{x}, \pi\right)$ vs wave vector $k_{x}$ for various values of hole doping. (c,d) Spin-spin correlation at a fixed and site-projected arrangement of holes along the rungs of the ladder. (c) is for four holes projected at the four red circles indicated. (d) is for eight holes projected at the eight red circles. Blue color lines represent AFM bonds, whereas FM bonds are represented with red color. These results were obtained using DMRG at $U / W=2.0$ and $J_{H} / U=0.25$, for cluster size $L=2 \times 12$.

netic ordering for different number of holes and interaction strength $U / W$, we calculate the spin-structure factor $S\left(k_{x}, k_{y}\right)=\frac{1}{L} \sum_{i, j} e^{\mathbf{k} \cdot \mathbf{r}_{i j}}\left\langle\mathbf{S}_{i} \cdot \mathbf{S}_{j}\right\rangle$ for cluster size $L=2 \times 12$ for the allowed set of wave vectors $\left(k_{x}, 0\right)$ and $\left(k_{x}, \pi\right)$.

Figure 2(a) contains the spin structure factor $S\left(k_{x}, 0\right)$ vs. wave vector $k_{x}$ at $U / W=2.0$ for various values of hole doping. At half filling $\left(N_{e}=48\right.$ in the $L=2 \times 12$ cluster), $S\left(k_{x}, 0\right)$ displays a sharp peak at $(\pi, 0)$, which is equivalent to AFM-order in the $x$-direction (leg) and FM-order along the $y$-direction (rung) of the ladder. This type of magnetic ordering is compatible with neutron experiments for $\mathrm{BaFe}_{2} \mathrm{~S}_{2}$ at ambient pressure [25]. Interestingly, increasing the number of holes, the peak of $S\left(q_{x}, 0\right)$ start splitting and the height of the peak decreases. This peak splitting at $(\pi, 0)$ increasing with the hole concentration indicates the appearance of spin incommensurability (IC) in the system [55]. This spin incommensurate spin fluctuations have been observed in the holeoverdoped iron-based high- $T_{c}$ compound $\mathrm{KFe}_{2} \mathrm{As}_{2}$ [27].
The decrease in intensity of the peak at $(\pi, 0)$ is due to the scrambling of spin-ordering by the holes, and also by the reduction in the number of electrons. Eventually, for large enough hole doping, $S\left(k_{x}, 0\right)$ displays no prominent ordering, while $S\left(k_{x}, \pi\right)$ shows a commensurate peak at $(\pi / 2, \pi)$, as shown in Fig. 2(b). At half-filling and for $U / W \gtrsim 1$ and $J_{H} / U=0.25$, the local spin moments are fully developed and approach their maximum value $S=1$ at each site for the two-orbital model used here (corresponding to magnetic moment 2.0 $\mu_{B}$ ) [40]. We find that the averaged spin-square expectation value $\left\langle S^{2}\right\rangle=\frac{1}{L} \sum_{i}\left\langle\mathbf{S}_{i} \cdot \mathbf{S}_{i}\right\rangle$ decreases linearly with hole doping $\left(N_{h}\right)$ [see inset of Fig. 3(b)], again suggesting the scrambling of local spin order with increasing holes $N_{h}$.

To better visualize in real space the spin IC and scrambling of spin-ordering when increasing the number of holes $N_{h}$, we have calculated the spin-spin correlations when the holes are projected into their most probable locations $[56,57]$. We found that the holes primarily reside in orbital $a$ due to the crystal-field splitting. For each number of holes, we focused on the configuration of holes with the highest probability in the wave function. In this case, to project out the dominant configuration of $N_{h}$ holes residing on orbital $a$, we use the projection operator [58] $P_{h a}(h)=P_{h a}\left(h_{1}\right) P_{h a}\left(h_{2}\right) \ldots P_{h a}\left(h_{m}\right)$ [with $P_{h a}(i)=c_{i a \uparrow} c_{i a \uparrow}^{\dagger} c_{i a \downarrow} c_{i a \downarrow}^{\dagger}$ acting as projectors on the ground state, namely finding the portion of the wave function where site $i$ and orbital $a$ are vacant (hole)].

After projecting the holes into their most probable locations, we calculate the local spin-spin correlations [40, 56, 57] $\left\langle\Psi \mid \mathbf{S}_{\mathbf{i}} a \cdot \mathbf{S}_{\mathbf{j}} a P_{h a} \Psi\right\rangle /\left\langle\Psi \mid P_{h a} \Psi\right\rangle$. In Ref. [40] the authors explored the spin-spin correlations for 1 and 2 holes at $U / W=2.0$, where they found robust AFM-correlations "across the holes". Here in Fig. 2(c) and Fig. 2(d), we analyze the spin-spin correlation for the cases of 4 and 8 holes, respectively, which are of more relevance for our focus on pairing. We find at $U / W=2$ that with 4 and 8 holes the spin AFM-correlation "across the holes" is still robust. The AFM-correlations in the leg direction and FM-correlations along-the rung, characteristic of the undoped regime, are mostly preserved in locations away from the hole positions. However, near the holes, AFM-correlations appear across the holes, leading to spin-IC tendencies and broadening of the $(\pi, 0)$ peak.

\section{B. Evolution of magnetic order with increasing $U / W$ towards the strong coupling regime}

Figure 3 displays the spin structure factors $S\left(k_{x}, 0\right)$ and $S\left(k_{x}, \pi\right)$ for different values of $U / W$ and at fixed number of holes $N_{h}=8$. In the weak-coupling limit $0.2 \lesssim U / W \lesssim 0.8$, the spin structure factor $S\left(k_{x}, 0\right)$ shows no prominent spin-ordering. In the range $0.8 \lesssim$ $U / W \lesssim 2.5, S\left(k_{x}, 0\right)$ shows incommensurate spin ordering, where we find short-range AFM-spin correlation 


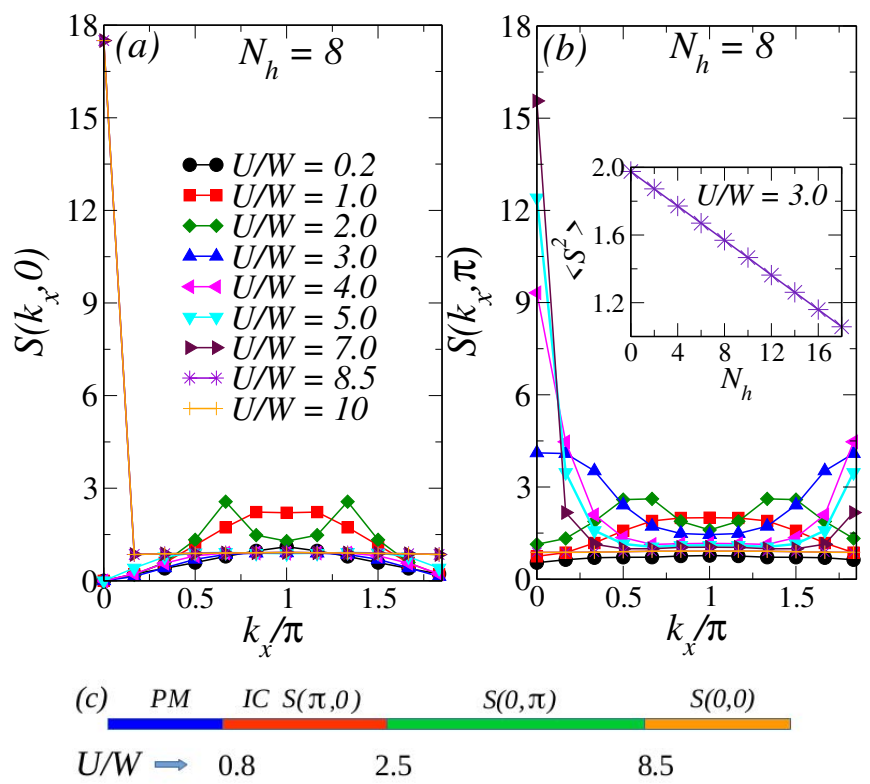

FIG. 3. Spin structure factor (a) $\mathrm{S}\left(k_{x}, 0\right)$ (b) $\mathrm{S}\left(k_{x}, \pi\right)$ vs wave vector $k_{x}$ for various values of interaction strength $U / W$ at fixed number of holes $N_{h}=8$. Inset shows site averaged $\left\langle S^{2}\right\rangle$ vs number of holes $N_{h}$ at $U / W=3.0$. (c) Sketch of the magnetic phase diagram at fixed $J_{H} / U=0.25$ and $N_{h}=8$.

along legs and FM-correlation along rungs of the ladder. Further increasing $U / W$, we find that the magnetic ordering evolves continuously and eventually ferromagnetic tendencies emerge along the leg direction. For interaction strength $2.5 \lesssim U / W \lesssim 8.5$ the $S(0, \pi)$ ordering, opposite to the previously discussed $S(\pi, 0)$ at smaller $U / W$, dominates [see Fig. 3(b)], which is equivalent to FM-spin ordering in the leg direction and AFM-spin ordering along the rung of the ladder.

Surprisingly, for $U / W \gtrsim 8.5$ the structure factor $S\left(k_{x}, 0\right)$ shows a strong peak at wave vector $(0,0)$ [Fig. 3(a)], indicating the appearance of ferromagnetic spin-order both along the leg and the rung directions of the ladder. As already explained, holes mainly reside on orbital $a$, while the population of orbital $b$ stays close to one electron/site (see Fig. 5). For this reason the orbital $b$ behaves as a localized spin $1 / 2$ system, while orbital $a$ provides delocalized holes. The movement (kinetic energy) of holes can improve if all spins aligned in the same direction [54], particularly at large $U / W$ because the Hund coupling is growing linearly with the Hubbard strength via $J_{H} / U=0.25$. This scenario is compatible with the double-exchange mechanism for manganites and results in ferromagnetic tendencies with increasing $U / W$. We find that for large doping the ferromagnetic tendency appears at smaller values of $U / W$ in comparison to the low-hole doping case [40].

The above described numerical results show that the magnetic order changes with increasing $U / W$. To un-
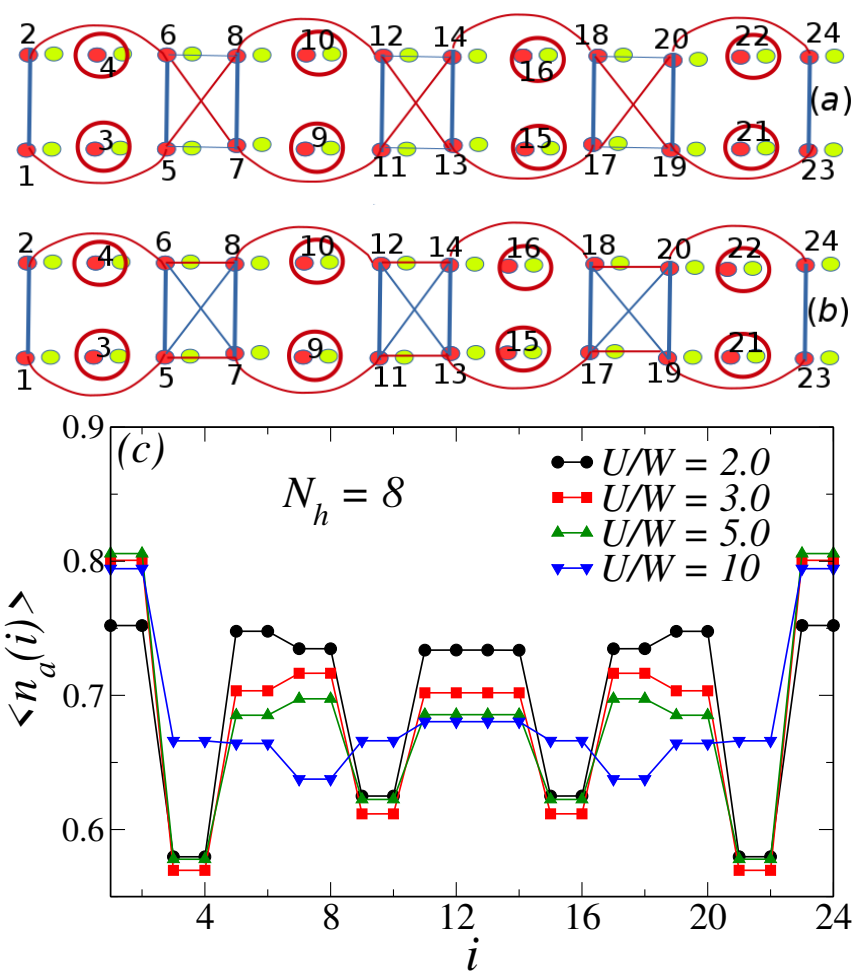

FIG. 4. (a,b) Spin-spin correlation at a fixed and siteprojected arrangement of $N_{h}=8$ holes along the rungs of the ladder. (a) at $U / W=3$, (b) at $U / W=5$. Blue color lines represent AFM bonds, whereas FM bonds are represented with red color. (c) Real-space density $\left\langle n_{a}(i)\right\rangle$ vs site index $i$ with $N_{h}=8$ holes and for various values of $U / W$.

derstand the connection between magnetic ordering and pairing of holes, in Fig. 4 we analyze the projected spin correlation $\left\langle\Psi \mid \mathbf{S}_{\mathbf{i}} a \cdot \mathbf{S}_{\mathbf{j}} a P_{h a} \Psi\right\rangle /\left\langle\Psi P_{h a} \Psi\right\rangle$ and the realspace charge density at orbital $a$ for different values of $U / W$. Figure 4(a) shows the projected spin correlations for $N_{h}=8$ at $U / W=3.0$. The FM magnetic order along the rung found at $U / W=2.0$ [Fig. 2(d)] now has changed to AFM order along the same rungs at $U / W=3.0$. Along the leg direction, at $U / W=2.0$ the order was fully AFM but at $U / W=3.0$ now there is a mixture: some bonds are FM and others are AFM. Once the FM vs AFM intensities are added, overall the leg magnetic ordering becomes ferromagnetic, consistent with the previously shown spin structure factor $S(0, \pi)$ [Fig. 3(b)]. Interestingly, at $U / W=5.0$ [Fig. 4(b)] the magnetic order along the legs switches completely to a FM order, whereas along the rung it is completely AFM order, i.e. the ordering is fully reversed as compared to $U / W=2.0$ where the rungs are FM and legs are AFM. For this reason at this coupling we find a peak at $(0, \pi)$ for the spin structure factor $S\left(k_{x}, \pi\right)$.

Figure 4(c) shows the real-space charge density of orbital $a$ for different values of $U / W$ with $N_{h}=8$ holes. It is clear from Fig. 4(c) that pairs still exist, and they are 
still primarily located along the rungs of the ladder, at all values of $U / W$ where there is a mixture of FM and AFM tendencies, namely whether favoring $(0, \pi)$ or $(\pi, 0)$ ordering. On the other hand, both in the limits of small $U / W$ with no magnetic order [Fig. 6(c)] and at very large $U / W$ such as $U / W=10.0$ [see Fig. 4(c), where the order is FM in both the rung and leg directions] we find that the density remains approximately uniform and there are no indications of pair formation because the density profile does not show four minima for the case of $N_{h}=8$.

\section{PAIRING TENDENCIES OF HOLES}
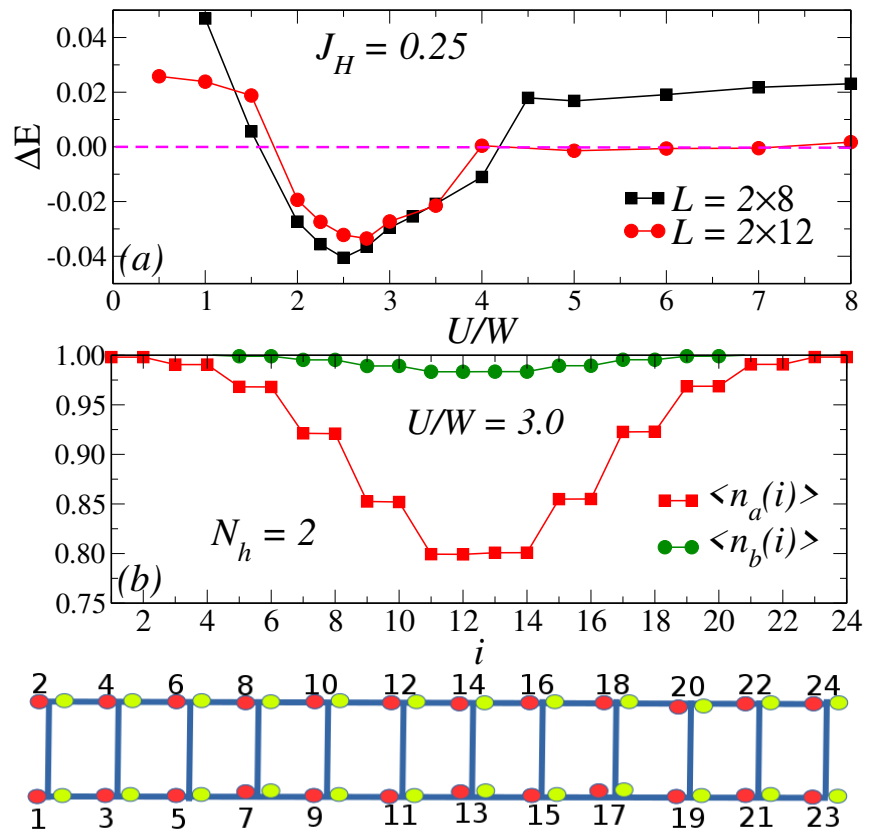

FIG. 5. (a) Binding energy $\Delta E$ vs. interaction strength $U / W$ at $J_{H} / U=0.25$ for cluster sizes $L=2 \times 8$ and $2 \times 12$ for comparison. (b) Real-space charge density $\left\langle n_{\alpha}\right\rangle$ vs site index $i$ for the case of $N_{h}=2$ holes and at $U / W=3$.0. Schematic representation of a two-leg ladder with the snake-like counting of sites appropriate for DMRG.

\section{A. Formation of holes pairs}

In this subsection, we investigate the pairing tendencies after the addition of holes to the undoped state. The formation of hole-pairs is a precursor of a pair-densitywave or of superconductivity $[40,54,55]$. Recent investigations indicate that in the high- $T_{c}$ cuprates, the region of pair-density-wave could become globally superconducting by a weak coupling between them [52].

In order to find the hole-pairing region as a function of interaction parameter $U / W$, we calculated the binding energy $\Delta E$. The binding energy of a pair of holes is defined as $\Delta E=E(N-2)+E(N)-2 E(N-1)$, where $E(N)$ is the ground state with $N$ electrons $[40,55]$. For a finite cluster, $\Delta<0$ is indicative of bound state of holes, while $\Delta>0$, or approximately zero, suggests two holes do not form a bound state. In Fig. 5(a), we show the binding energy for two cluster sizes $L=2 \times 8$ and $L=2 \times 12$ vs. interaction parameter $U / W$ and at fixed $J_{H} / U=0.25$. Interestingly, the binding energies $\Delta$ become negative in the region $1.6 \lesssim U / W \lesssim 4.0$, indicate of formation of stable bound pairs of holes in this regime. The similarity among the values of binding energies for both cluster sizes $L=2 \times 8$, and $2 \times 12$ indicate small finite size effects in the bnding region. Note that in the bulk limit, when there is no pairing the binding energy should be zero because the energy of 2 holes is the same as 2 times the energy of 1 hole, measured with respect to the undoped ground state. The case $2 \times 12$ already shows this behavior in a broad range of robust $U / W$. At weak coupling, where size effects are often larger than in strong coupling, the tendency increasing $L$ is in the right direction: $2 \times 12$ has smaller binding energy than $2 \times 8$ before true binding occurs.

To further verify the pairing of holes, we analyze the real-space charge density $\left\langle n_{\alpha}\right\rangle$ with two holes at $U / W=$ 3.0. As shown in Fig. 5(b), the occupancy of orbital $b$ is close to one, while there is a deep minimum in the density of orbital $a$. This indicates that holes reside mainly at orbital $a$. This charge profile is also compatible with a bound state, as opposed to two unbounded holes where two minima would be expected. The real-space density $\left\langle n_{a}(i)\right\rangle$ of orbital $a$, also suggest that binding of holes occurs near the center of the cluster, either along the same rung or along the plaquette diagonals, consistent with the negative value of binding energy at $U / W=3.0$.

To understand the pairing tendencies for more than two holes, in Figs. 6(a) and (b), we plot the real-space charge densities for each orbital with $N_{h}=4$ and $N_{h}=8$ holes at $U / W=3.0$. The densities of orbital $b$ remain approximately one, and holes reside mainly on orbital $a$. These tendencies resemble the orbital selective Mott phase discussed extensively in recent literature related to ladders and chains [59-63], at doping $N_{h}=4$ and $N_{h}=8$.

For orbital $a$ and with 4 holes Fig. 6(a), there exist two minima in the real-space charge density, compatible with two bound pairs of holes. These four holes are primarily located along the rungs with site indexes $[i=7,8]$ and $[i=17,18]$ in the snake-geometry convention. For the case of $N_{h}=8$ holes there exist four minima [see Fig. 6(b)], and these minima are also located with the highest chances along the rungs of the ladder. Figure 6(c) shows the density of orbital $a$ and $b$ at lower values of the interaction $U / W=0.2$, for the case of $N_{h}=8$ holes. In this weak coupling example, the density of orbitals, both $\left\langle n_{a}\right\rangle$ and $\left\langle n_{b}\right\rangle$, takes nearly uniform values. There are no minima in the density profile, compatible with no bound 

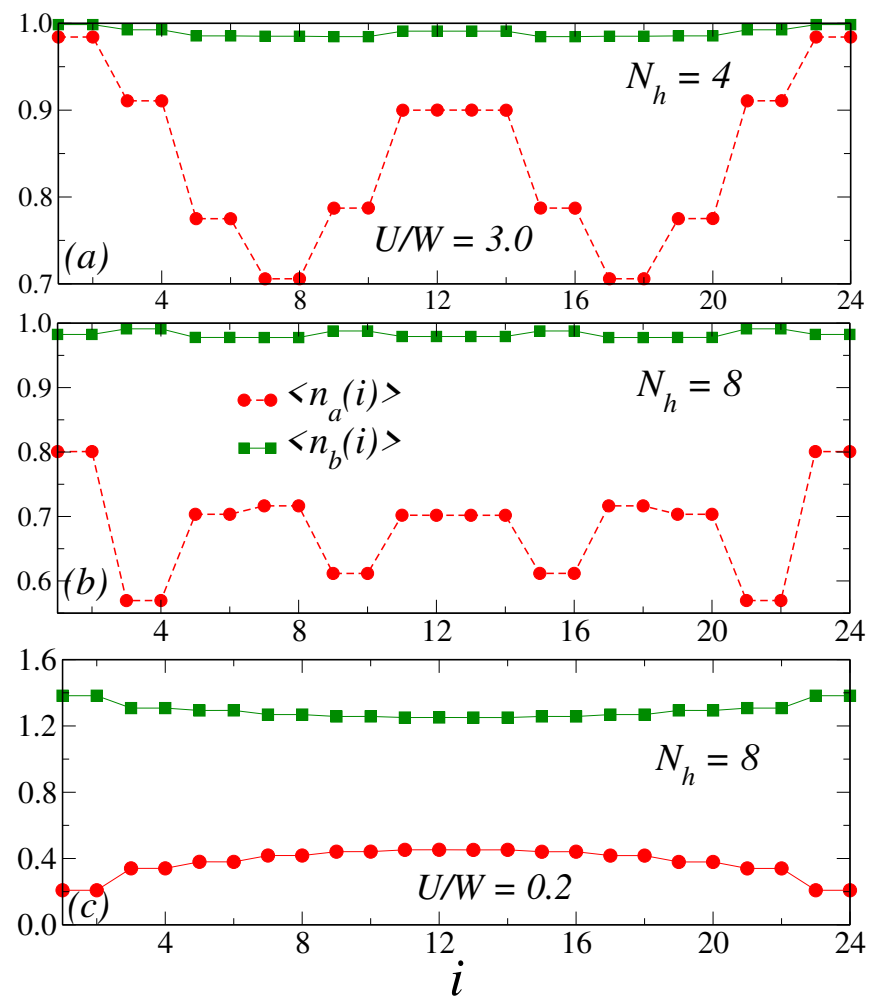

FIG. 6. Real-space density profile at $U / W=3.0$ (a) $N_{h}=$ 4 holes and for (b) $N_{h}=8$.holes. (c) Real-space density profile at $U / W=0.2$ with $N_{h}=8$ holes. These numerical calculations were performed using DMRG for a cluster size $L=2 \times 12$ and at fixed $J_{H} / U=0.25$.

pairs formation in this range of $U / W$. The results above suggest that for larger hole doping, holes form bound pairs along the rungs of the ladder and these holes reside mainly on orbital $a$, result compatible with our previous investigations.

\section{B. Superconducting Correlations}

Superconductivity involves pairing of holes, as in Cooper pairs, followed by the development of coherence among pairs in the system forming a condensate [48, 64]. In this last subsection, we show computational evidence compatible with pairing of holes in some narrow regions of doping. We certainly do not exclude that instead of a coherent superconductor, we instead have a pair-density wave. But, as already explained, recent neutron scattering results for two-dimensional cuprates are compatible with the presence of a pair-density in ladders in the stripe regime, followed by phase coherence induced by the coupling among ladders [52]. Either way, we believe our results are evidence of superconductivity tendencies in our models of iron-based ladders.

First let us analyze the pair-correlations for various values of hole dopings. We calculate the pair-pair cor-
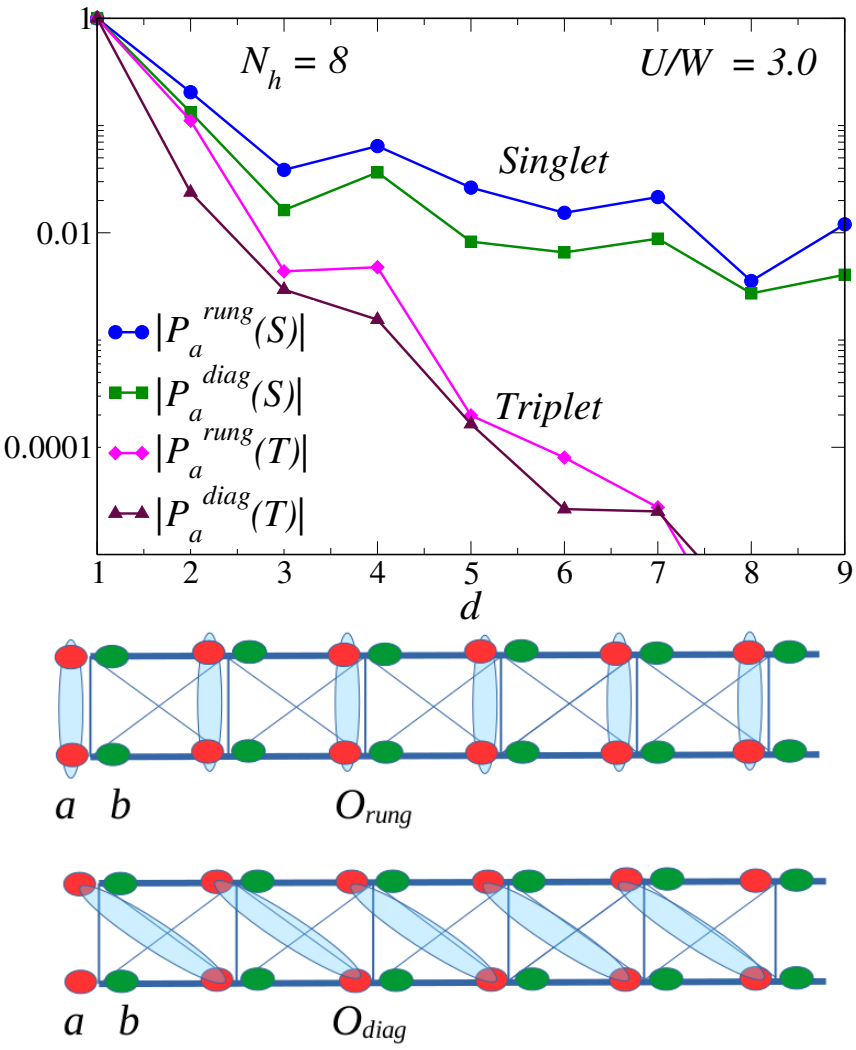

FIG. 7. Singlet and triplet pair-correlations, $P_{a}^{\text {rung }}(S)$ and $P_{a}^{\text {rung }}(T)$, calculated along the vertical rung, whereas $P_{a}^{\text {diag }}(S)$ and $P_{a}^{\text {diag }}(T)$ are calculated along diagonal of the ladder, as indicated in the sketch at the bottom of the figure. All results obtained at $U / W=3.0$ and $J_{H} / U=0.25$ for a size cluster $L=2 \times 12$. Bottom: schematic diagrams of vertical $O_{\text {rung }}$ and diagonal $O_{\text {diag }}$ pairing operators involving orbital a.

relations at a fixed value of the interaction strength $U / W=3.0$, and for cluster size $L=2 \times 12$. For our model with two-leg ladder geometry and two orbitals ( $a$ and $b$ ) at each site, we could have a variety of pair operators to use in the pair-pair correlations. Early investigations suggest that properly selecting the pair operator enhances the signal for superconductivity. However, that task is complicated. For simplicity, here we have focused on intraorbital nearest-neighbor sites pairing-operators (certainly, the presence of local on-site Hubbard repulsion $U$ and $U^{\prime}$ renders the possibility of electron on-site pairing negligible, opposite to the negative $U$ Hubbard model) [55]. As discussed in previous subsections, the holes reside mainly on orbital $a$ and form pairs along the rungs and digonals of the ladder. For this reason, we calculate the correlation functions focusing only on orbital $a$ and along both the rung and plaquette diagonal directions of the ladder. We have also checked the paircorrelations along the leg directions, but we found they decay at a much faster rate compared to all other correlations. To avoid colliding with one another, the optimal 
case for the holes in the pair is to have one hole at one leg, and the other hole at the other leg.

As a consequence, in order to examine the nature of the dominating superconducting correlation, we analyzed the singlet and triplet pair correlation functions [44, 65] along the vertical and diagonal rungs (see schematic shown in Fig. 4). The nearest-neighbor singlet pairing operator for orbital $a$ along the vertical rung is defined as

$$
O_{\text {rung, } S}^{\dagger}(i, a)=\frac{1}{\sqrt{2}}\left[c_{i, a, 1, \uparrow}^{\dagger} c_{i, a, 2, \downarrow}^{\dagger}-c_{i, a, 1, \downarrow}^{\dagger} c_{i, a, 2, \uparrow}^{\dagger}\right],
$$

where $i$ is the site index, while 1 and 2 are the leg index. The nearest-neighbor triplet pairing operator for orbital $a$ along the vertical rung is

$$
O_{\text {rung,T }}^{\dagger}(i, a)=\frac{1}{\sqrt{2}}\left[c_{i, a, 1, \uparrow}^{\dagger} c_{i, a, 2, \downarrow}^{\dagger}+c_{i, a, 1, \downarrow}^{\dagger} c_{i, a, 2, \uparrow}^{\dagger}\right] .
$$

The singlet pairing operator for orbital $a$ along the ladder plaquette diagonal is

$$
O_{\text {diag, } S}^{\dagger}(i, a)=\frac{1}{\sqrt{2}}\left[c_{i, a, 2, \uparrow}^{\dagger} c_{i+1, a, 1, \downarrow}^{\dagger}-c_{i, a, 2, \downarrow}^{\dagger} c_{i+1, a, 1, \uparrow}^{\dagger}\right] .
$$

Finally, the intraorbital spin triplet pairing operator along the ladder plaquette diagonal is

$$
O_{\text {diag, } T}^{\dagger}(i, a)=\frac{1}{\sqrt{2}}\left[c_{i, a, 2, \uparrow}^{\dagger} c_{i+1, a, 1, \downarrow}^{\dagger}+c_{i, a, 2, \downarrow}^{\dagger} c_{i+1, a, 1, \uparrow}^{\dagger}\right]
$$

Using these pairing operators, we have calculate the averaged superconducting pair-pair correlations for orbital $a$ defined as

$$
P_{a}(d)=\frac{1}{N_{d}} \sum_{i}\left\langle O_{a}^{\dagger}(i) O_{a}(i+d)\right\rangle,
$$

where $d=|i-j|$ is the distance along the leg of the ladder, and $N_{d}$ is the number of equal-distant pairs from site $i$. In other words, we consider all the distances within our clusters, properly normalized.

In Fig. 7, we show the spin-singlet pair correlations $\left[P_{a}^{\text {rung }}(S)\right.$ and $\left.P_{a}^{\text {diag }}(S)\right]$ and triplet pair correlations $\left[P_{a}^{r u n g}(T)\right.$ and $\left.P_{a}^{\text {diag }}(T)\right]$ for orbital $a$ with $N_{h}=8$ holes. While calculating the pair correlations, we have discarded the first and last rungs, to reduce boundary effects. We find that the spin singlet correlation along the rung $P_{a}^{\text {rung }}(S)$ is the most dominating pair correlation. This is to be expected due to that fact that holes form a bounded pair along the vertical rung with the highest probability. The spin-singlet correlation along the diagonal rung $P_{a}^{\text {diag }}(S)$ also decays similarly, although with slightly less amplitude, becoming the second most dominating pair-pair correlation. On the other hand, the spin-triplet pair correlations $P_{a}^{\text {rung }}(T)$ and $P_{a}^{\text {diag }}(T)$ decay exponentially (i.e. approximately linearly in the logarithmic scale used). These numerical results show that

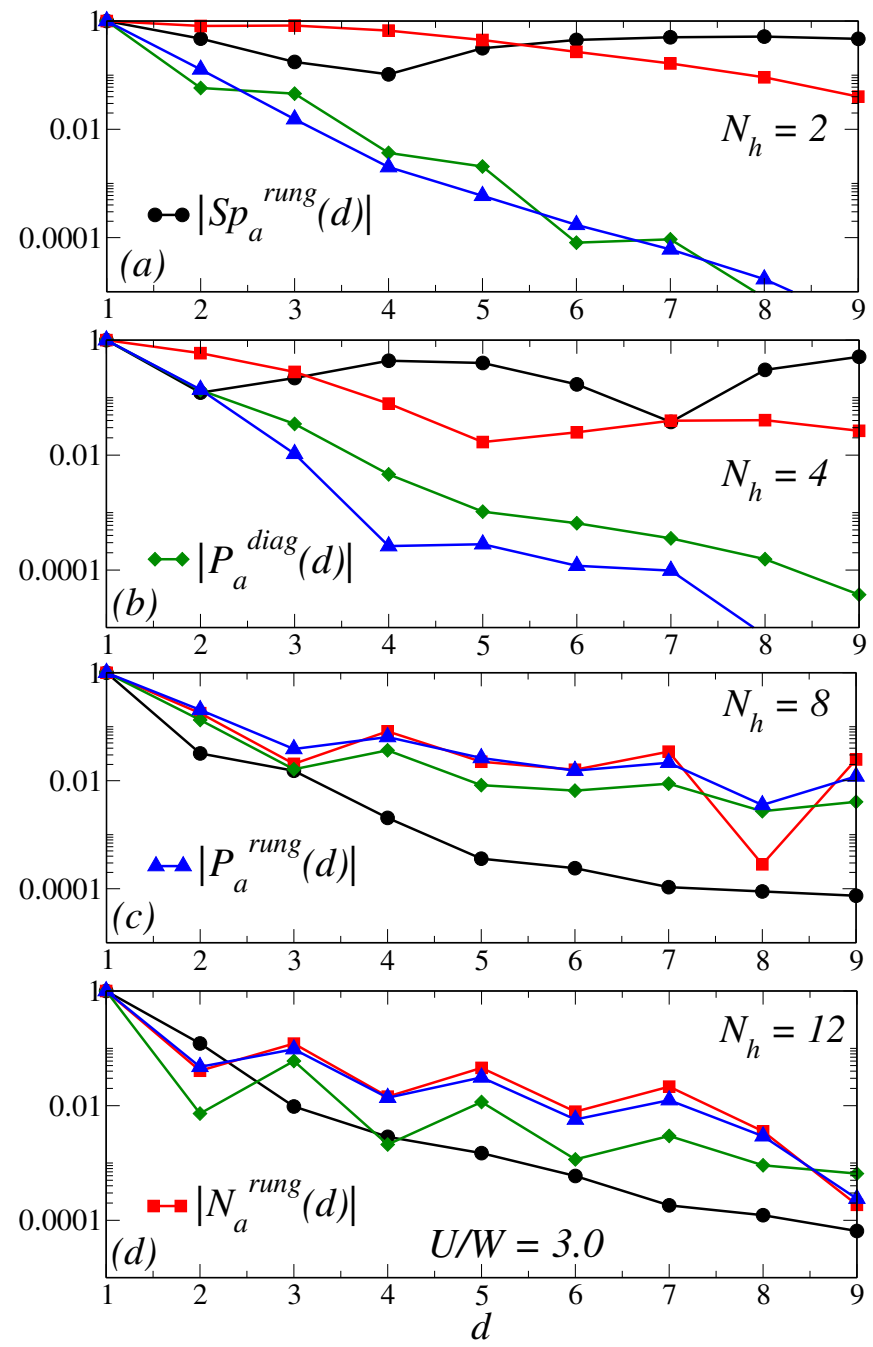

FIG. 8. Comparison of spin-spin $S p_{a}^{\text {rung }}(d)$, charge-charge $N_{a}^{\text {rung }}(d)$, and singlet pair-pair $P_{a}^{\text {rung }}(d)$ and $P_{a}^{\text {diag }}(d)$ correlations. The various panels correspond to (a) $N_{h}=2$, (b) $N_{h}=4$, (c) $N_{h}=8$, and (d) $N_{h}=12$ holes, all at $U / W=3.0$ and $J_{H} / U=0.25$ and using a system size $L=2 \times 12$.

the pair-correlation is robust in the spin-singlet channel and primarily along the rungs of the ladder.

In a quasi-one-dimensional system, the slowest decaying correlation functions at long distance determine the dominant type of ordering. It is expected that once inter chain or ladder couplings are turned on, this slowest decaying channel will become dominant. In order to compare the pair-pair correlations with spin and charge correlations for orbital $a$, we have calculated the rungspin and rung-charge correlations [66]. We define the combined rung spin as $\mathbf{S}_{a, i}=\left(\mathbf{S}_{a, i, 1}+\mathbf{S}_{a, i, 2}\right)$, where 1 and 2 refer to the leg index of the ladder. The averaged run spin-correlation then becomes $S p_{a}(d)=$ $\frac{1}{N_{d}} \sum_{i}\left\langle\mathbf{S}_{a, i} \cdot \mathbf{S}_{a, i+d}\right\rangle$. For the rung charge we use the definition $N_{a, i}=\left(N_{a, i, 1}+N_{a, i, 2}\right)$, and the charge-charge correlations for orbital $a$ are calculated via $N_{a}(d)=$ 
$\frac{1}{N_{d}} \sum_{i}\left\langle N_{a, i} N_{a, i+d}\right\rangle-\left\langle N_{a, i}\right\rangle\left\langle N_{a, i+d}\right\rangle$. For spin and charge correlations also we have discarded the first and last rungs of the ladder, to reduce boundary effects.

Figure 8 contains the comparison of pair-pair correlations vs. rung-spin and rung-charge correlation, for various values of hole dopings, at $U / W=3.0$. For low doping $N_{h}=2$ and $N_{h}=4$, the spin correlation $S p_{a}^{r u n g}(d)$ clearly dominates over all other correlations [see Fig. 8(a) and Fig. 8(b)]. This is understandable: even with pair formation, the spin channel has not been fully scrambled and coherence over long distances remains. A typical high- $T_{c}$ superconductor remains antiferromagnetic over a finite range of doping starting in the undoped parent compound. For this reason, the singlet pair correlations $P_{a}^{\text {rung }}(d)$ and $P_{a}^{\text {diag }}(d)$ decay with much faster rate for lower dopings.

Interestingly, when we increase the hole doping to $N_{h}=8$ and 10 (the latter not shown), we find that the rung-singlet pair correlation $P_{a}^{\text {rung }}(d)$ starts competing with the charge correlation $N_{a}^{\text {rung }}(d)$. At large doping, the spin-correlation $S p_{a}^{r u n g}(d)$ decays with faster rate due to scrambling of spin-ordering in the presence of holes [see Fig. 8(c) and (d)]. With further increase in the hole concentration the rung-charge correlation $N_{a}^{\text {rung }}(d)$ dominates over all other correlations and the singlet correlation also decreases in magnitude [Fig. 8(d)].

Remarkably, as shown in Fig. 8c, the rung-singlet pair correlation $P_{a}^{\text {rung }}(d)$ decays with similar rate as the rungcharge correlation $N_{a}^{r u n g}(d)$ for $N_{h}=8$ holes. A similar decay rate of rung-singlet pair correlation with charge correlations suggest the existence of a pair-density wave state at $U / W=3.0[50-52]$. The pair-density wave is an exotic state, with interwined charge density and superconducting orders. We believe superconductivity can be achieved by weak inter-ladder Josephson coupling [52], which can transform the one-dimensional pair-densitywave state to a two-dimensional coherent superconducting state. In a recent experiment it has been shown that the superconductivity found in $\mathrm{BaFe}_{2} \mathrm{~S}_{3}$ has a bulk origin [25]. Also they found that increasing pressure leads to an increase in the inter-ladder transfer of $\mathrm{Fe} 3 d$ electrons, and as a result the system turns more metallic [25]. In the case of an array of $\mathrm{Cu}$-based two-leg doped ladders, namely with one active orbital, it was found that with intra-ladder coupling leads to a superconducting state $[67,68]$.

\section{Effect of interaction and Hund's coupling}

In Fig. 9(a), we show a comparison of the rung-singlet pair correlation $P_{a}^{\text {rung }}(d)$ at $U / W=3.0$ and for three different values of Hund's coupling $J_{H} / U$, with $N_{h}=8$ holes. As shown in Fig. 9(a), the singlet pair correlation $P_{a}^{\text {rung }}(d)$ decays with a faster rate at $J_{H} / U=0.1$. On the other hand, the singlet pair correlation $P_{a}^{\text {rung }}(d)$ is

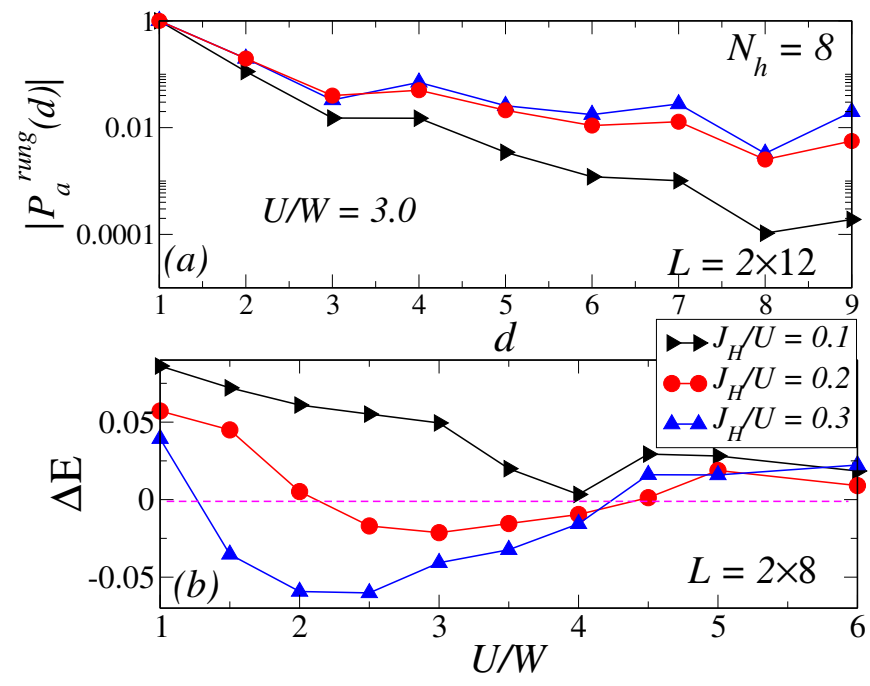

FIG. 9. (a) Rung singlet pair-pair $P_{a}^{\text {rung }}(d)$ at $U / W=3.0$ with $N_{h}=8$ holes, for different values of $J_{H} / U$. (b) Binding energy $\Delta E$ vs. interaction strength $U / W$ for different values of Hund's coupling $J_{H} / U$, using a cluster size $L=2 \times 8$.

enhanced by increasing $J_{H} / U$. The increase in magnitude of $P_{a}^{\text {rung }}(d)$ is clearly consistent with the results for the binding energy (i.e. $\Delta E$ become negative for $\left.J_{H} / U \gtrsim 0.15\right)$.

We have also studied the effect of Hund's coupling $J_{H} / U$ on the bindings of holes. In Fig. 9(b), we display the binding energy $\Delta E$ vs. interaction strength $U / W$ for three different values of $J_{H} / U$. At $J_{H} / U=0.1$ binding energy remain positive or close to zero for all values of $U / W$, indicating no binding occurs for lower values of Hund's coupling. However, for $J_{H} / U=0.2$ and 0.3 the binding energy becomes negative in a wide range of $U / W$. The (negative) value of $\Delta E$ increases as $J_{H} / U$ increases. These results clearly show that the Hund's coupling $J_{H} / U$ plays an important role in inducing pairing and in the enhancement of singlet-pair correlations.

\section{Pairing tendencies with an alternative set of hopping parameters}

Let us examine now the binding of holes using an alternative set of hopping parameters. The new set of hopping parameters at pressure $P=12.36 \mathrm{GPa}$ was derived also using first-principles density functional theory band structure calculations [based on the Vienna ab initio simulation package (VASP) code [69-71] and the maximally localized Wannier function as implemented in the WANNIER90 code [72, 73]]. To obtain the hopping matrices, we only focus on the band around the Fermi level and considered the hopping matrix with distance up to $\sqrt{2}$ Fe-Fe. Thus, modifications on the hopping matrix were performed accordingly to render the tight binding band structure to fit better the $a b$ initio results. It must be 

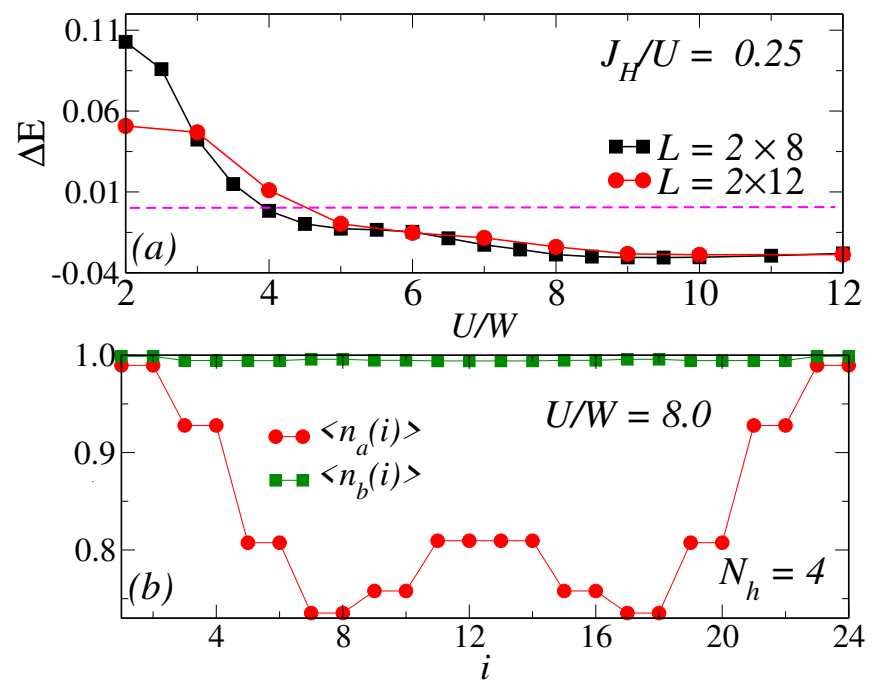

FIG. 10. Results using an alternative set of hoppings. (a) Binding energy $\Delta E$ vs. interaction strength $U / W$ at $J_{H} / U=$ 0.25 for cluster sizes $L=2 \times 8$ and $2 \times 12$. (b) Real-space charge density $\left\langle n_{\alpha}\right\rangle$ vs. site index $i$ with $N_{h}=4$ holes and at $U / W=8.0$.

clarified that the technical need to constraint the matching between band structure and tight-binding results using only two orbitals (for practical reasons, because a DMRG study of a two-leg three-orbital ladder would be too costly) renders the set of hoppings not unique. Thus, it is convenient to analyze what physics is obtained with alternative hopping sets.

Thus, here we will repeat part of the previous results using the following $2 \times 2$ hopping matrix between sites $i$ and $i+\hat{x}$ and along the legs of the ladder $t_{\gamma, \gamma^{\prime}}^{x}$ defined by (in eV units):

$$
t_{\gamma, \gamma^{\prime}}^{x}=\left[\begin{array}{cc}
-0.65 & +0.25 \\
-0.25 & +0.216
\end{array}\right]
$$

where $\gamma$ is the orbital index for site $i$ and $\gamma^{\prime}$ for $i+\hat{x}$, as before. $t_{\gamma, \gamma^{\prime}}^{y}$ is the $2 \times 2$ hopping matrix along the vertical rung direction:

$$
t_{\gamma, \gamma^{\prime}}^{y}=\left[\begin{array}{cc}
-0.10 & 0.00 \\
0.00 & +0.181
\end{array}\right]
$$

The hoppings $t^{x+y}$ and $t^{x-y}$ are $2 \times 2$ hopping matrices along the plaquette diagonals of the ladder:

$$
t_{\gamma, \gamma^{\prime}}^{x+y}=t_{\gamma, \gamma^{\prime}}^{x-y}=\left[\begin{array}{cc}
+0.05 & -0.322 \\
+0.322 & +0.084
\end{array}\right]
$$

The crystal fields $\Delta_{\gamma}$ at $P=12.36$ GPa for each orbital are in this case $\Delta_{a}=0.70 \mathrm{eV}$ and $\Delta_{b}=-0.297 \mathrm{eV}$. The kinetic energy bandwidth is $W=3.238 \mathrm{eV}$.

Figure 10(a) shows the binding energy $\Delta E$ vs. interaction strength $U / W$, using this new hopping parameters for cluster sizes $L=2 \times 8$ and $L=2 \times 12$ at $J_{H} / U=0.25$.
With the new set of hoppings the binding energy takes negative values at larger values of $U / W$, compared to the older hopping parameters. Both sets of hoppings lead to a similar magnitude of $\Delta E \approx-0.04 \mathrm{eV}$ in the binding region, in spite of the different $U / W$ range for the binding. Clearly, pairing of holes is possible for both set of hopping parameters.

The binding of holes is further confirmed by calculating the real-space density $\left\langle n_{a}(i)\right\rangle$ for different number of holes $N_{h}=4,6,8$, where we find 2 minima for 4 holes, 3 minima for 6 holes, and 4 minima for 8 holes. Figure 10(b) shows the real-space density plots for orbital $a$ and $b$. The density of orbital $b$ remains close to one, while there are two minima in the density $\left\langle n_{a}(i)\right\rangle$ of orbital $a$, showing the formation of two hole pairs at $U / W=8.0$. The pairing of holes along the rung and negative values of binding energy, namely the qualitative similarities with the other set, suggest that the new hoppings will likely also develop a pair density wave or directly a superconductor adding couplings among ladders.

\section{CONCLUSIONS}

In this publication, we have studied the doped twoorbital Hubbard model for two-leg ladders with hopping parameters corresponding to the compound $\mathrm{BaFe}_{2} \mathrm{~S}_{3}$. Using DMRG calculations for cluster sizes up to $L=$ $2 \times 12$, we have investigated magnetic and pairing tendencies for various doping strengths. We find different type of magnetic ordering when increasing the interaction strength $U / W$ and hole concentrations. For moderate values of interaction strength, the system shows incommensurate magnetic ordering, involving short range AFM-correlation along the leg and FM-correlation along the rung direction, as in the compound at ambient pressure that in our case corresponds to the undoped limit.

Remarkably, in the presence of holes and with increase in $U / W$, the AFM-order along the leg direction changes to FM-ordering, whereas FM-order along rungs changes to AFM-order. For even larger values of $U / W$, FM-order appears along both leg and rung directions. Interestingly, we find a robust evidence of pairing of holes. The binding energy becomes negative (pair formation) for wide ranges of interaction parameter $U / W$. The real-space density profile of orbital $a$ shows clear indication of pairing of holes along the rungs of the ladder. Moreover, we have presented a comparison of rung-spin, rung-charge, and pair-pair correlations. At lower doping concentration, we find the rung-spin (magnetic) correlation dominates over other correlations. For moderate values of doping of holes we find an exotic pair-density wave state, where the rung-singlet pair correlation and rung-charge correlation coexist. Furthermore, we have shown that the Hund's coupling plays an important role in the pairing of holes. In addition, we have also shown that the qualitative as- 
pects of the pairing of holes are similar employing two sets of hopping matrices, suggesting that our study is generic and survives in a wide range in parameter space.

\section{ACKNOWLEDGMENTS}

The work of B.P, R. S, L.-F.L., and E.D. was supported by the U.S. Department of Energy (DOE), Office of Science, Basic Energy Sciences (BES), Materials Sciences and Engineering Division. G.A. was partially supported by the Center for Nanophase Materials Sciences, which is a U.S. DOE Office of Science User Facility, and by the Scientific Discovery through Advanced Computing (SciDAC) program funded by U.S. DOE, Office of Science, Advanced Scientific Computing Research and Basic Energy Sciences, Division of Materials Sciences and Engineering. Validation and some computer runs were conducted at the Center for Nanophase Materials Sciences, which is a DOE Office of Science User Facility.

[1] Y. Kamihara, H. Hiramatsu, M. Hirano, R. Kawamura, H. Yanagi, T. Kamiya, and H. Hosono, J. Am. Chem. Soc. 128, 10012 (2006). https://doi.org/10. 1021/ja063355c

[2] Y. Kamihara, T. Watanabe, M. Hirano, and H. Hosono, J. Am. Chem. Soc. 130, 3296 (2008). https://doi.org/ 10.1021/ja800073m

[3] H. Takahashi, K. Igawa, K. Arii, Y. Kamihara, M. Hirano, and H. Hosono, Nature (London) 453, 376 (2008). https://doi.org/10.1038/nature06972

[4] G. F. Chen, Z. Li, D. Wu, G. Li, W. Z. Hu, J. Dong, P. Zheng, J. L. Luo, and N. L. Wang, Phys. Rev. Lett. 100, 247002 (2008). https://doi.org/10.1103/ PhysRevLett.100.247002

[5] H. Luetkens, H.-H. Klauss, M. Kraken, F. J. Litterst, T. Dellmann, R. Klingeler, C. Hess, R. Khasanov, A. Amato, C. Baines, M. Kosmala, O. J. Schumann, M. Braden, J. Hamann-Borrero, N. Leps, A. Kondrat, G. Behr, J. Werner and B. Buchner, Nature Mater. 8, 305 (2009). https://doi .org/10.1038/nmat2397

[6] M. H. Fang, H. M. Pham, B. Qian, T. J. Liu, E. K. Vehstedt, Y. Liu, L. Spinu, and Z. Q. Mao, Phys. Rev. B 78, 224503 (2008). https://doi.org/10.1103/ PhysRevB.78.224503

[7] Fong-Chi Hsu, Jiu-Yong Luo, Kuo-Wei Yeh, Ta-Kun Chen, Tzu-Wen Huang, Phillip M. Wu, Yong-Chi Lee, Yi-Lin Huang, Yan-Yi Chu, Der-Chung Yan, and MawKuen Wu, Proc. Natl Acad. Sci. USA 105, 14262 (2008). https://doi.org/10.1073/pnas.0807325105

[8] D. C. Johnston, Adv. Phys. 59, 803 (2010). https:// doi.org/10.1080/00018732.2010.513480

[9] G. R. Stewart, Rev. Mod. Phys. 83, 1589 (2011). https : //doi.org/10.1103/RevModPhys.83.1589

[10] J. Guo, S. Jin, G. Wang, S. Wang, K. Zhu, T. Zhou, M. He, and X. Chen, Phys. Rev. B 82, 180520(R) (2010). https://doi.org/10.1103/PhysRevB.82.180520
[11] Ming-Hu Fang, Hang-Dong Wang, Chi-Heng Dong, ZuJuan Li, Chun-Mu Feng, J. Chen and H. Q. Yuan, Europhys. Lett. 94, 27009 (2011). https://doi.org/10. 1209/0295-5075/94/27009

[12] B. Wei, Li Guan-Nan, H. Qing-Zhen, C. Gen-Fu, H. JunBao, W. Du-Ming, M. A. Green, Q. Yi-Ming, L. Jian-Lin, W. Mei-Mei Chin. Phys. Lett. 30, 027402 (2013). http: //cpl.iphy.ac.cn/10.1088/0256-307X/30/2/027402

[13] E. Dagotto, Rev. Mod. Phys. 85, 849 (2013). https:// doi.org/10.1103/RevModPhys .85.849

[14] W. Li, S. Dong, C. Fang, and J. Hu, Phys. Rev. B 85, 100407(R) (2012) https://doi.org/10.1103/PhysRevB. 85.100407

[15] I. I. Mazin, Nature 464, 183 (2010). https://doi.org/ 10.1038/nphys 2438

[16] P. J. Hirschfeld, M. M. Korshunov, and I. I. Mazin , Rep. Prog. Phys. 74, 124508 (2011). https://doi.org/ 10.1088/0034-4885/74/12/124508

[17] J. Paglione and R. L. Greene, Nature Physics 6, 645(2010). https://doi.org/10.1038/nphys 1759

[18] K. Kuroki, S. Onari, R. Arita, H. Usui, Y. Tanaka, H. Kontani, and H. Aoki, Phys. Rev. Lett. 101, 087004 (2008). https://doi.org/10.1103/ PhysRevLett.101.087004

[19] D. Liu, W. Zhang, D. Mou, J. He, Y. Ou, Q. Wang, Z. Li, L. Wang, L. Zhao, and S. He, Y. Peng, Xu Liu, C. Chen, Li Yu, G. Liu, X. Dong, J. Zhang, C. Chen, Z. Xu, J. Hu, Xi Chen, X. Ma, Q. Xue, Nat. Commun. 3, 931 (2012). https://doi.org/10.1038/ncomms1946

[20] F. Bondino, E. Magnano, M. Malvestuto, F. Parmigiani, M. A. McGuire, A. S. Sefat, B. C. Sales, R. Jin, D. Mandrus, E. W. Plummer, D. J. Singh, and N. Mannella, Phys. Rev. Lett. 101, 267001 (2008). https://doi.org/ 10.1103/PhysRevLett.101.267001

[21] P. C. Dai, J. P. Hu, and E. Dagotto, Nat. Phys. 8, 709 (2012). https://doi.org/10.1038/nphys2438

[22] B. Wei, H. Qing-Zhen, C. Gen-Fu, W. Du-Ming, He Jun-Bao and Q. Yi-Ming, Chinese Phys. Lett. 28, 086104 (2011). https://doi.org/10.1088/0256-307X/ 28/8/086104

[23] F. Ye, S. Chi, Wei Bao, X. F. Wang, J. J. Ying, X. H. Chen, H. D. Wang, C. H. Dong, and Minghu Fang, Phys. Rev. Lett. 107, 137003 (2011). https://doi.org/ 10.1103/PhysRevLett.107.137003

[24] T. Yamauchi, Y. Hirata, Y. Ueda, and K. Ohgushi, Phys. Rev. Lett. 115, 246402 (2015). https://doi.org/10. 1103/PhysRevLett.115.246402

[25] H. Takahashi, A. Sugimoto, Y. Nambu, T. Yamauchi, Y. Hirata, T. Kawakami, M. Avdeev, K. Matsubayashi, F. Du, C. Kawashima, H. Soeda, S. Nakano, Y. Uwatoko, Y. Ueda, T. J. Sato, and K. Ohgushi, Nat. Mater. 14, 1008 (2015). https://doi.org/10.1038/nmat4351

[26] S. Avci, O. Chmaissem, E. A. Goremychkin, S. Rosenkranz, J.-P. Castellan, D. Y. Chung, I. S. Todorov, J. A. Schlueter, H. Claus, M. G. Kanatzidis, A. Daoud-Aladine, D. Khalyavin, and R. Osborn, Phys. Rev. B 83, 172503 (2011). https://doi.org/10.1103/ PhysRevB.83.172503

[27] C. H. Lee, K. Kihou, H. Kawano-Furukawa, T. Saito, A. Iyo, H. Eisaki, H. Fukazawa, Y. Kohori, K. Suzuki, H. Usui, K. Kuroki, and K. Yamada, Phys. Rev. Lett. 106, 067003 (2011). https://doi.org/10.1103/ PhysRevLett.106.067003 
[28] M. Rotter, M. Tegel, and D. Johrendt Phys. Rev. Lett. 101, 107006 (2008). https://doi.org/10.1103/ PhysRevLett.101.107006

[29] J. M. Caron, J. R. Neilson, D. C. Miller, A. Llobet, and T. M. McQueen, Phys. Rev. B 84, 180409(R) (2011). https://doi.org/10.1103/PhysRevB.84.180409

[30] B. Saparov, S. Calder, B. Sipos, H. Cao, S. Chi, D. J. Singh, A. D. Christianson, M. D. Lumsden, and A. S. Sefat, Phys. Rev. B 84, 245132 (2011). https://doi. org/10.1103/PhysRevB.84.245132

[31] J. M. Caron, J. R. Neilson, D. C. Miller, K. Arpino, A. Llobet, and T. M. McQueen, Phys. Rev. B 85, 180405(R) (2012). https://doi.org/10.1103/ PhysRevB.85.180405

[32] S. Chi, Y. Uwatoko, H. Cao, Y. Hirata, K. Hashizume, T. Aoyama, and K. Ohgushi, Phys. Rev. Lett. 117, 047003 (2016). https://doi.org/10.1103/ PhysRevLett.117.047003

[33] M. Mourigal, Shan Wu, M. B. Stone, J. R. Neilson, J. M. Caron, T. M. McQueen, and C. L. Broholm, Phys. Rev. Lett. 115, 047401 (2015). https://doi.org/10. 1103/PhysRevLett.115.047401

[34] Y. Zhang, L.-F. Lin, A. Moreo, S. Dong, and E. Dagotto, Phys. Rev. B 100, 184419 (2019). https://doi.org/10. 1103/PhysRevB.100.184419

[35] Y. Zhang, L.-F. Lin, A. Moreo, S. Dong, and E. Dagotto, Phys. Rev. B 101, 144417 (2020). https://doi.org/10. 1103/PhysRevB.101.144417

[36] N. D. Patel, N. Kaushal, A. Nocera, G. Alvarez and E. Dagotto, npj Quantum Materials 5, 27 (2020). https: //doi.org/10.1038/s41535-020-0228-2

[37] Q. Luo, A. Nicholson, J. Rincón, S. Liang, J. Riera, G. Alvarez, L. Wang, Wei Ku, G. D. Samolyuk, A. Moreo, and E. Dagotto, Phys. Rev. B 87, 024404 (2013). https: //doi.org/10.1103/PhysRevB.87.024404

[38] N.D. Patel, A. Nocera, G. Alvarez, A. Moreo, S. Johnston, E. Dagotto, Commun Phys 2, 64 (2019). https: //doi.org/10.1038/s42005-019-0155-3

[39] B. Pandey, Ling-Fang Lin, R. Soni, N. Kaushal, J. Herbrych, G. Alvarez, and E. Dagotto, Phys. Rev. B 102, 035149 (2020). https://doi.org/10.1103/PhysRevB. 102.035149

[40] N. D. Patel, A. Nocera, G. Alvarez, R. Arita, A. Moreo, and E. Dagotto, Phys. Rev. B 94, 075119 (2016). https: //doi.org/10.1103/PhysRevB.94.075119

[41] H. Lei, H. Ryu, A. I. Frenkel, and C. Petrovic, Phys. Rev. B 84, 214511 (2011). https://doi.org/10.1103/ PhysRevB.84.214511

[42] Y. Nambu, K. Ohgushi, S. Suzuki, F. Du, M. Avdeev, Y. Uwatoko, K. Munakata, H. Fukazawa, S. Chi, Y. Ueda, and T. J. Sato, Phys. Rev. B 85, 064413 (2012). https: //doi.org/10.1103/PhysRevB.85.064413

[43] J. M. Caron, J. R. Neilson, D. C. Miller, K. Arpino, A. Llobet, and T. M. McQueen, Phys. Rev. B 85, 180405(R) (2012). https://doi.org/10.1103/ PhysRevB.85.180405

[44] E. Dagotto, J. A. Riera, and D. J. Scalapino, Phys. Rev. B 45, 5744(R) (1992). https://doi.org/10.1103/ PhysRevB.45.5744

[45] E. Dagotto and T. M. Rice, Science 271, 618 (1996). https://doi.org/10.1126/science.271.5249.618

[46] M. Uehara, T. Nagata, J. Akimitsu, H. Takahashi, N. Môri, and K. Kinoshita, J. Phys. Soc. Jpn. 65, 2764 (1996). https://doi.org/10.1143/JPSJ.65.2764
[47] T. Osafune, N. Motoyama, H. Eisaki, and S. Uchida, Phys. Rev. Lett. 78, 1980 (1997). https://doi.org/10. 1103/PhysRevLett.78.1980

[48] E. Dagotto, Rev. Mod. Phys. 66, 763 (1994). https:// doi.org/10.1103/RevModPhys.66.763

[49] S. R. White, Phys. Rev. Lett. 69, 2863 (1992). https: //doi.org/10.1103/PhysRevLett.69.2863

[50] E. Fradkin, S. A. Kivelson, and J. M. Tranquada, Rev. Mod. Phys. 87, 457 (2015). https://doi.org/10.1103/ RevModPhys .87.457

[51] D. F. Agterberg, J.C. Séamus Davis, S. D. Edkins, E. Fradkin, D. J. Van Harlingen, S. A. Kivelson, P. A. Lee, L. Radzihovsky, J. M. Tranquada, and Y. Wang, Annual Review of Condensed Matter Physics 11, Agterberg, 231 (2020). https://doi.org/10.1146/ annurev-conmatphys-031119-050711

[52] J. M. Tranquada, arXiv:2102.02257 (2021), and references therein. https://arxiv.org/abs/2102.02257

[53] G. Alvarez, Comput. Phys. Commun.180, 1572 (2009). https://doi.org/10.1016/j.cpc.2009.02.016

[54] J. C. Xavier, G. Alvarez, A. Moreo, and E. Dagotto, Phys. Rev. B 81, 085106 (2010). https://doi.org/10. 1103/PhysRevB.81.085106

[55] N. D. Patel, A. Nocera, G. Alvarez, A. Moreo, and E. Dagotto, Phys. Rev. B 96, 024520 (2017). https://doi. org/10.1103/PhysRevB.96.024520

[56] G. B. Martins, C. Gazza, J. C. Xavier, A. Feiguin, and E. Dagotto ,Phys. Rev. Lett. 84, 5844 (2000). https: //doi.org/10.1103/PhysRevLett.84.5844

[57] G. B. Martins, J. C. Xavier, C. Gazza, M. Vojta, and E. Dagotto, Phys. Rev. B 63, 014414 (2000). https://doi. org/10.1103/PhysRevB.63.014414

[58] S. R. White and D. J. Scalapino, Phys. Rev. B 55, 6504 (1997) https://doi.org/10.1103/PhysRevB.55.6504

[59] J. Rincón, A. Moreo, G. Alvarez, and E. Dagotto, Phys. Rev. Lett. 112, 106405 (2014). https://doi.org/10. 1103/PhysRevLett.112.106405

[60] J. Herbrych, N. Kaushal, A. Nocera, G. Alvarez, A. Moreo, and E. Dagotto, Nature Communications 9, 3736 (2018). https://doi.org/10.1038/ s41467-018-06181-6

[61] J. Herbrych, J. Heverhagen, N. D. Patel, G. Alvarez, M. Daghofer, A. Moreo, and E. Dagotto, Phys. Rev. Lett. 123027203 (2019). https://doi.org/10.1103/ PhysRevLett.123.027203

[62] J. Herbrych, J. Heverhagen, G. Alvarez, M. Daghofer, A. Moreo, and E. Dagotto, PNAS 117, 16226 (2020). https://doi.org/10.1073/pnas. 2001141117

[63] J. Herbrych, G. Alvarez, A. Moreo, and E. Dagotto, Phys. Rev. B. 102, 115134 (2020). https://doi.org/ 10.1103/PhysRevB.102.115134

[64] V. J. Emery, S. A. Kivelson, and O. Zachar, Phys. Rev. B 56, 6120 (1997). https://doi.org/10.1103/PhysRevB. 56.6120

[65] T. Shirakawa, S. Nishimoto, and Y. Ohta, Phys. Rev. B 77, 224510 (2008). https://doi.org/10.1103/ PhysRevB.77.224510

[66] A. Nocera, N. D. Patel, E. Dagotto, and G. Alvarez, Phys. Rev. B 96, 205120 (2017). https://doi.org/10. 1103/PhysRevB.96.205120

[67] E. Arrigoni, E. Fradkin, and S. A. Kivelson, Phys. Rev. B 69, 214519 (2004). https://doi.org/10.1103/ PhysRevB.69.214519 
[68] S. R. White and D. J. Scalapino, Phys. Rev. Lett. 80, 1272 (1998). https://doi.org/10.1103/PhysRevLett. 80.1272

[69] G. Kresse and D. Joubert, Phys. Rev. B 59, 1758 (1999). https ://doi.org/10.1103/PhysRevB.59.1758

[70] P. E. Böchl, Phys. Rev. B 50, 17953 (1994). https:// doi.org/10.1103/PhysRevB.50.17953
[71] O. P. Perdew, A. Ruzsinszky, G. I. Csonka, O. A. Vydrov, G. E. Scuseria, L. A. Constantin, X. Zhou, and K. Burke Phys. Rev. Lett. 100, 136406 (2008). https://doi.org/ 10.1103/PhysRevLett.100.136406

[72] N. Marzari and D. Vanderbilt, Phys. Rev. B 56, 12847 (1997). https://doi.org/10.1103/PhysRevB.56.12847

[73] A. A. Mosto, J. R. Yates, Y. S. Lee, I. Souza,i D. Vanderbilt, and N. Marzari, Comput. Phys. Commun. 178, 685 (2007). https://doi.org/10.1016/j.cpc.2007.11.016 